\title{
Exposure to ambient particulate matter and biomass burning during pregnancy: associations with birth weight in Thailand
}

\author{
William Mueller $\mathbb{D}^{1} \cdot$ Kraichat Tantrakarnapa $^{2} \cdot$ Helinor Jane Johnston $^{3} \cdot$ Miranda Loh $^{1} \cdot$ Susanne Steinle $^{1}$. \\ Sotiris Vardoulakis ${ }^{1,4} \cdot$ John W. Cherrie ${ }^{1,3}$
}

Received: 22 June 2020 / Revised: 12 December 2020 / Accepted: 18 January 2021 / Published online: 18 February 2021

(c) The Author(s) 2021. This article is published with open access

\begin{abstract}
Background There is a growing evidence that exposure to ambient particulate air pollution during pregnancy is associated with adverse birth outcomes, including reduced birth weight (BW). The objective of this study was to quantify associations between BW and exposure to particulate matter (PM) and biomass burning during pregnancy in Thailand.

Methods We collected hourly ambient air pollutant data from ground-based monitors (PM with diameter of $<10 \mu \mathrm{m}\left[\mathrm{PM}_{10}\right]$, Ozone $\left[\mathrm{O}_{3}\right]$, and nitrogen dioxide $\left[\mathrm{NO}_{2}\right]$ ), biomass burning from satellite remote sensing data, and individual birth weight data during 2015-2018. We performed a semi-ecological analysis to evaluate the association between mean trimester exposure to air pollutants and biomass burning with BW and low-birth weight (LBW) $(<2500 \mathrm{~g})$, adjusting for gestation age, sex, previous pregnancies, mother's age, heat index, season, year, gaseous pollutant concentrations, and province. We examined potential effect modification of $\mathrm{PM}_{10}$ and biomass burning exposures by sex.

Results There were 83,931 eligible births with a mean pregnancy $\mathrm{PM}_{10}$ exposure of $39.7 \mu \mathrm{g} / \mathrm{m}^{3}$ (standard deviation [SD] $=$ 7.7). The entire pregnancy exposure was associated with reduced BW both for $\mathrm{PM}_{10}\left(-6.81 \mathrm{~g}\right.$ per $10 \mu \mathrm{g} / \mathrm{m}^{3}$ increase in $\mathrm{PM}_{10}$ $[95 \% \mathrm{CI}=-12.52$ to -1.10$])$ and biomass burning $\left(-6.34 \mathrm{~g}\right.$ per $1 \mathrm{SD}$ increase in fires $/ \mathrm{km}^{2}[95 \% \mathrm{CI}=-11.35$ to -1.34$\left.]\right)$ only after adjustment for $\mathrm{NO}_{2}$. In contrast with these findings, a reduced odds ratio (OR) of LBW was associated with PM 10 exposure only in trimesters one and two, with no relationship across the entire pregnancy period. Associations with biomass burning were limited to increased ORs of LBW with exposure in trimester three, but only for male births.

Conclusion Based on our results, we encourage further investigation of air pollution, biomass burning and BW in Thailand and other low-income and middle-income countries.
\end{abstract}

Supplementary information The online version contains supplementary material available at https://doi.org/10.1038/s41370021-00295-8.

$\triangle$ John W. Cherrie

j.cherrie@hw.ac.uk

1 Institute of Occupational Medicine, Edinburgh, UK

2 Department of Social and Environmental Medicine, Faculty of Tropical Medicine, Mahidol University, Bangkok, Thailand

3 School of Engineering and Physical Sciences, Institute of Biological Chemistry, Biophysics and Bioengineering, Heriot Watt University, Edinburgh, UK

4 National Centre for Epidemiology and Population Health, Research School of Population Health, Australian National University, Canberra, ACT, Australia

\section{Introduction}

There is a growing evidence that exposure to ambient particulate air pollution during pregnancy is associated with adverse birth outcomes, including preterm birth [1], small for gestational age [2], and reduced birth weight (BW) $[3,4]$. Determining the causality of these relationships is critical, since any adverse associations at birth may have life-long implications for health [5].

Steinle et al. [6] identified available systematic reviews and meta-analyses to evaluate the plausibility of a concentration-response function between ambient particulate matter (PM) and BW and low birth weight (LBW) (i.e., $<2500$ g [7]). Although the identified meta-analyses found large heterogeneity between individual studies, there emerged adverse associations between continuous and binary $\mathrm{BW}$ measures and exposures to $\mathrm{PM}$ with aerodynamic diameters of $<10 \mu \mathrm{m}\left(\mathrm{PM}_{10}\right)$ and $<2.5 \mu \mathrm{m}\left(\mathrm{PM}_{2.5}\right)$; 
for example, odds ratios (ORs) for LBW ranged from 1.01 (95\% CI $=0.96-1.08)$ [8] to $1.05(95 \% \mathrm{CI}=1.02-1.07)$ [9] per $10 \mu \mathrm{g} / \mathrm{m}^{3}$ of $\mathrm{PM}_{10}$.

To support and possibly explain these epidemiological findings, evidence from in vivo studies has shown that ambient PM, ultrafine carbon black, and diesel exhaust particles can cause reproductive and developmental toxicity following pulmonary exposure (reviewed by [10, 11]). The exact mechanism(s) underlying PM toxicity to the developing embryo/foetus is yet to be elucidated, but could involve particle translocation across the placenta, the ability of particles to cause altered placental function (e.g., due to the activation of inflammation and oxidative stress), and/or the presence of circulating inflammatory mediators produced in the mother following exposure of the lungs to PM (reviewed by [12]). In addition to PM, other ambient air pollutants, such as ozone $\left(\mathrm{O}_{3}\right)$ and nitrogen dioxide $\left(\mathrm{NO}_{2}\right)$, have been associated with reduced $\mathrm{BW}$, possibly acting via similar mechanisms of oxidative stress and inflammation [13, 14].

The majority of the published epidemiological studies that investigate air pollution and birth outcomes originate from North America, Europe, and other higher income regions. In the review by Steinle et al. [6], only about $8 \%$ of the contributing studies in the meta-analyses were from Asia. Furthermore, these previous Asian studies have predominantly taken place in temperate regions (e.g., China, South Korea); BW in tropical nations may be additionally adversely affected by malaria and the presence of other infections or parasites occurring during pregnancy [15]. Also, as far as we are aware, none of the Asian studies were undertaken in settings where the population is routinely exposed to PM emissions from extensive burning of crop residues or forest, which can represent a significant PM source during these periods $[16,17]$. The few existing studies of BW and maternal exposure to biomass burning have produced mixed results [18]. These knowledge gaps represent major limitations in the existing evidence base, particularly as large populations in Asia are exposed to high levels of PM from urban sources and biomass burning.

Our aim was to investigate in Thailand the relationship between continuous BW and LBW and cumulative exposure to $\mathrm{PM}_{10}$ air pollution and biomass burning during pregnancy. To help identify any sensitive windows, we assessed these exposures in the presence of gaseous air pollutants for the entire gestation length and also during potential critical periods, thus allowing for variation in pollution concentrations and sources occurring throughout the year. This study is part of the larger research project to study the effects of air pollution in Thailand: Thailand Air Pollution Health Impact Assessment.

\section{Methods}

\section{Study setting}

Thailand is situated in the tropics, with distinct wet (May-October), hot (March-May), and dry (November-February) seasons; with the exception of the north, temperatures are in excess of $30^{\circ} \mathrm{C}$ for most of the year [19]. It is a fast developing upper middle-income country with a population of almost 70 million people. Thailand covers a land area of $513,000 \mathrm{~km}^{2}$, with around $41 \%$ for agriculture and $37 \%$ for forest [20]. Despite ongoing efforts by the government to reduce agricultural burning of crop residues, it is still widespread in Thailand: during 2019, there were $~ 35,000$ fires detected [21]. Most of the fires in Thailand occur between December and April each year, and as a consequence, ambient particulate air pollution concentrations are significantly elevated during those months [22]. In addition to biomass burning, emissions from industry, traffic, and power plants are the main contributors of PM, carbon monoxide $(\mathrm{CO})$ and nitrogen oxides $\left(\mathrm{NO}_{x}\right)$, which can be precursors for the formation of $\mathrm{O}_{3}$ [23].

More than $99 \%$ of births are registered in Thailand [24]. Thailand is committed to providing universal healthcare for the population, funded from general taxation; as part of this provision, all births are attended by an experienced healthcare professional and $90.8 \%$ of expectant mothers attend four or more visits to antenatal care [25, 26].

\section{Birth outcome data}

We obtained anonymised data on individual births during the years 2010-2018 for all Thailand provinces, except Bangkok (see below), from the Ministry of Public Health. The following variables were included in this dataset: infant sex, gravidity (i.e., number of previous pregnancies), BW (g), gestational age (in weeks), maternal age, date of birth, a binary indicator for any congenital anomalies, and International Classification of Diseases (ICD) coding for birth outcomes; however, the latter variable was mostly missing (>90\%) and was therefore excluded. Some variables contained a high proportion $(>50 \%)$ of missing data in the years 2010-2014, and total birth counts were lower in 2013 and 2014 compared to that of the other years with no apparent explanation. Therefore, based on the higher quality of the birth data in the most recent years of the dataset, we defined the study period to be 1 January 2015 to 30 April 2018.

We also collected for the years 2014-2018 anonymised individual birth data for Bangkok from the Bangkok Metropolitan Administration. This dataset contained variables for date of birth, sex of the baby, BW (g), and ICD coding to signify information such as single/multiple births and any 
Fig. 1 Map of the study area in Thailand, showing the included provinces (shaded) and locations of air pollution ground monitors.

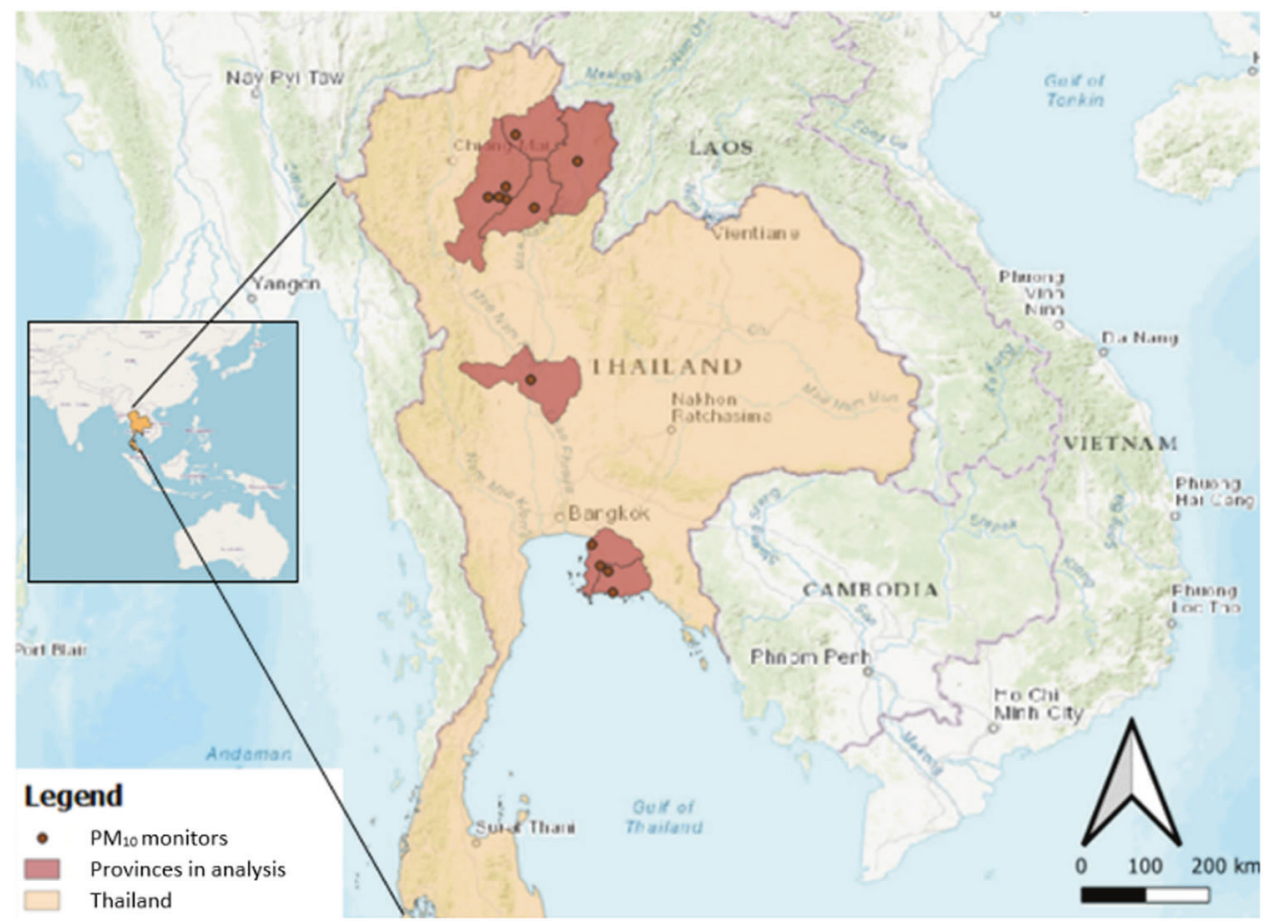

pregnancy complications; however, gravidity, maternal age, and gestation age in weeks were not included in this dataset. Due to the omission of these important risk factors for BW [27], particularly given the typically more modest magnitude of risks associated with air pollution, we focussed our analysis on the Thai provinces with more complete data and thus excluded the Bangkok data from analysis.

\section{Exposure data}

\section{Air pollutants}

The Thai Pollution Control Department (PCD) manages a network of ground-based stations to monitor air pollutants throughout the country. We collected hourly air quality data from the PCD network to identify all monitors during the study period with sufficient $\mathrm{PM}_{10}, \mathrm{PM}_{2.5}, \mathrm{NO}_{2}$, and $\mathrm{O}_{3}$ data. Mean daily data from a given monitor were considered to be sufficient if $\geq 75 \%$ of measurements (i.e., $\geq 18 \mathrm{~h}$ ) of each pollutant were available on a given day [28] and $\geq 75 \%$ of such days were available during the study period. We selected as the main exposure $\mathrm{PM}_{10}$ concentrations instead of $\mathrm{PM}_{2.5}$, since only three monitors included sufficient $\mathrm{PM}_{2.5}$ monitoring data (compared to 13 monitors with $\mathrm{PM}_{10}$ data). The mean daily temperature $\left({ }^{\circ} \mathrm{C}\right)$ and relative humidity $(\%)$ data, which were included with the air pollutant data from each station, were calculated in the same manner. For $\mathrm{O}_{3}$, maximum daily values of the 8 -h rolling average were calculated, where six or more hours of data were available [29].

Each province was assigned the mean daily value from monitors with sufficient data; the average value was calculated if data were available from multiple monitors on the same day. These daily data were used to generate average trimester exposures over three 90 -day periods preceding the date of birth, where at least 68 days (i.e., $\geq 75 \%$ ) with data were available in each trimester. Exposure over the entire pregnancy was calculated as the mean of the three trimesters. Only those monitors with sufficient data (i.e., $<25 \%$ missing data) for all pollutants (i.e., $\mathrm{PM}_{10}, \mathrm{NO}_{2}, \mathrm{O}_{3}$ ) were eligible for analysis $(n=12$; Table S1).

\section{Biomass burning}

As an indicator for biomass burning, we obtained satellite remote sensing data from NASA's Visible Infra-red Imaging Radiometer Suite sensor on the daily number of fires in Thailand during the study period at a spatial resolution of $375 \mathrm{~m}$ [30]. We excluded any fires demarcated as low confidence and retained only those categorised as vegetation fires. To standardise the data, we summed the number of fires in each province for each trimester and the entire pregnancy period, as described above, and divided by the area of the province; these values were then converted to $\mathrm{z}$ scores for analysis.

\section{Statistical analysis}

We used a semi-ecological study design to develop linear and logistic regression models to assess, respectively, the association between exposure to province-level averages of particulate air pollution and biomass burning during pregnancy with two health outcomes from individual birth data: 
a continuous measure $(\mathrm{g})$ and a binary outcome for LBW (defined as $<2500 \mathrm{~g}$ ). We included in the models covariates which have been previously shown to be important predictors of BW and for which we had data: gestation age in weeks, sex [31], gravida (continuous), and mother's age (cubic spline with three knots based on lower BIC values) [27]. In Thailand, PM levels demonstrate strong seasonal trends; instead of adjusting for season, but to incorporate seasonal differences that might impact birth outcomes [32], we calculated an average heat index (HI) for each trimester based on temperature and relative humidity using Steadman's formula, as presented in Anderson et al. [33]. We also accounted for potential unmeasured regional differences and long-term trends in BW by including covariates for province and year, respectively; this would help model differences in BW according to the timing, and thus exposure, of gestation. Only normal term pregnancies were included (i.e., gestation age of 37-41 weeks) with a maternal age of 14-60 years and a maximum of 20 previous pregnancies. Analysis was restricted to BWs of 1000-5200 g (i.e., \pm 5 standard deviations above and below the mean [34]). Analysis was conducted using those births with complete data in those provinces with $<25 \%$ missing air pollution data ( $n=7$; Fig. 1).

Three classes of models are presented for each outcome: (1) model 1 unadjusted, (2) model $1+$ all above covariates, (3) model $2+$ other individual air pollutants. Regression coefficients were rescaled for all pollutants to represent changes per $10 \mu \mathrm{g} / \mathrm{m}^{3} ; \mathrm{O}_{3}$ and $\mathrm{NO}_{2}$ were converted to $\mu \mathrm{g} / \mathrm{m}^{3}$ from ppb using conversion factors of 1.96 and 1.88 , respectively [35]. We adjusted for gaseous air pollutants in the models of $\mathrm{PM}_{10}$ and biomass burning if spearman rank correlations were $<0.7$; thus we included $\mathrm{NO}_{2}$ and excluded $\mathrm{O}_{3}$. Regression models were run with either exposure in all three trimesters [36] or an average of the entire pregnancy. Residuals were examined using histograms and Q-Q plots. Additional analyses were run to examine effect modification in $\mathrm{PM}_{10}$ and biomass burning exposures with sex. To assess the robustness of the BW data, we used tests of proportion to determine if the last digit (or two) of BWs were more likely to be rounded to the nearest 10 and $100 \mathrm{~g}$ (i.e., different from $10 \%$ or $1 \%$ of all births). We used QGIS (v3.10.1-A Coruña) for geospatial analysis with biomass burning data and performed statistical analysis using Stata (v15.1).

\section{Results}

During the study period (2015-2018), there were 83,931 eligible births (out of a total of 158,$457 ; 53.0 \%$ ) with complete exposure and birth data in the study area. The
Table 1 Descriptive characteristics of the study sample $(N=83,931)$.

\begin{tabular}{|c|c|}
\hline Characteristics & Mean (SD) or $n(\%)$ \\
\hline Birth weight (g) & $3112(408.5)$ \\
\hline \multicolumn{2}{|c|}{ Low birth weight $(<2500 \mathrm{~g})$} \\
\hline Yes & $4413(5.3 \%)$ \\
\hline No & $79,518(94.7 \%)$ \\
\hline \multicolumn{2}{|l|}{$\mathrm{PM}_{10}\left(\mu \mathrm{g} / \mathrm{m}^{3}\right)$} \\
\hline Trimester 1 & $40.2(18.5)$ \\
\hline Trimester 2 & $38.8(17.2)$ \\
\hline Trimester 3 & $40.2(17.3)$ \\
\hline Entire pregnancy & $39.7(7.7)$ \\
\hline \multicolumn{2}{|l|}{$\mathrm{O}_{3}\left(\mu \mathrm{g} / \mathrm{m}^{3}\right)$} \\
\hline Trimester 1 & $78.9(26.6)$ \\
\hline Trimester 2 & $76.8(23.9)$ \\
\hline Trimester 3 & $78.7(24.6)$ \\
\hline Entire pregnancy & $78.1(12.8)$ \\
\hline \multicolumn{2}{|l|}{$\mathrm{NO}_{2}\left(\mu \mathrm{g} / \mathrm{m}^{3}\right)$} \\
\hline Trimester 1 & $16.4(7.0)$ \\
\hline Trimester 2 & $16.4(7.2)$ \\
\hline Trimester 3 & $16.8(7.7)$ \\
\hline Entire pregnancy & $16.5(5.8)$ \\
\hline \multicolumn{2}{|c|}{ Biomass burning (fires $/ \mathrm{km}^{2}$ ) } \\
\hline Trimester 1 & $0.06(0.11)$ \\
\hline Trimester 2 & $0.05(0.09)$ \\
\hline Trimester 3 & $0.05(0.09)$ \\
\hline Entire pregnancy & $0.15(0.15)$ \\
\hline \multicolumn{2}{|l|}{ Sex } \\
\hline Male & $43,343(51.6 \%)$ \\
\hline Female & $40,588(48.4 \%)$ \\
\hline \multicolumn{2}{|l|}{ Gestation } \\
\hline 37 weeks & $11,496(13.7 \%)$ \\
\hline 38 weeks & $27,425(32.7 \%)$ \\
\hline 39 weeks & $24,009(28.6 \%)$ \\
\hline 40 weeks & $17,560(20.9 \%)$ \\
\hline 41 weeks & $3441(4.1 \%)$ \\
\hline Maternal age (years) & $26.9(6.5)$ \\
\hline Gravidity & $1.9(1.0)$ \\
\hline \multicolumn{2}{|l|}{ Heat index } \\
\hline Trimester 1 & $31.1(3.5)$ \\
\hline Trimester 2 & $30.6(3.3)$ \\
\hline Trimester 3 & $30.2(3.3)$ \\
\hline Entire pregnancy & $30.6(2.5)$ \\
\hline \multicolumn{2}{|l|}{ Year } \\
\hline 2015 & $14,865(17.7 \%)$ \\
\hline 2016 & $23,856(28.4 \%)$ \\
\hline 2017 & $37,161(44.3 \%)$ \\
\hline 2018 & $8049(9.6 \%)$ \\
\hline
\end{tabular}


Table 1 (continued)

\begin{tabular}{lc}
\hline Characteristics & Mean (SD) or $n(\%)$ \\
\hline Province & \\
Chon Buri & $17,500(20.9 \%)$ \\
Rayong & $26,149(31.2 \%)$ \\
Lampang & $14,186(16.9 \%)$ \\
Phrae & $1684(2.0 \%)$ \\
Nan & $6265(7.5 \%)$ \\
Phayao & $5713(6.8 \%)$ \\
Nakhon Sawan & $12,434(14.8 \%)$ \\
\hline
\end{tabular}

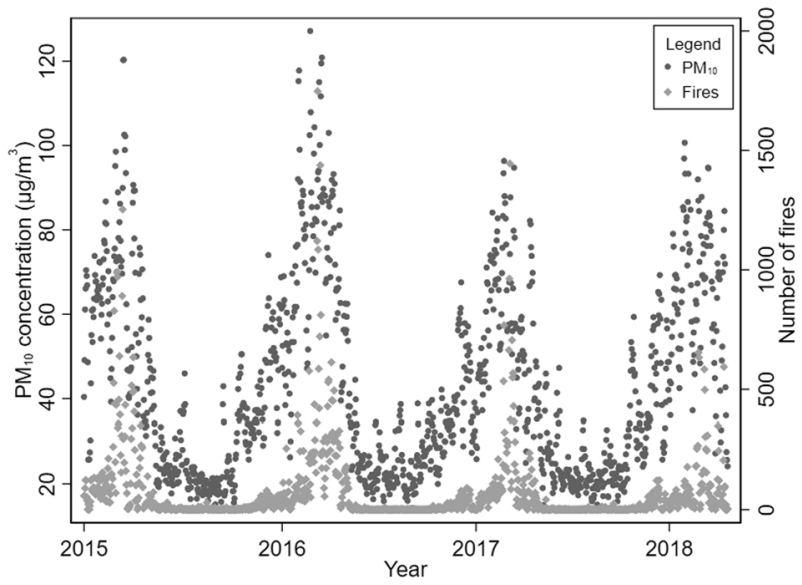

Fig. 2 The daily mean $\mathrm{PM}_{10}$ concentrations and total number of fires across the study area.

mean $\mathrm{BW}$ was $3112 \mathrm{~g}(\mathrm{SD}=408.5)$, with $5.3 \%$ of births categorised as LBW. Nearly one third (32.7\%) of the births occurred in the 38th week, and just under half $(48.4 \%)$ of babies born were female. The average mother's age was 26.9 years, with an average of about two (1.9) previous pregnancies (Table 1). There was strong evidence of rounded BWs: $77.7 \%$ (vs $10.0 \%$ expected) of recorded weights had ' 0 ' as the last digit and $10.3 \%$ (vs $1.0 \%$ expected) as the last two digits $(p<0.001$ in each instance).

There was a clear seasonal trend in $\mathrm{PM}_{10}$ concentrations and the number of daily fires (see Fig. 2). Mean exposure concentrations in each trimester were very similar within each pollutant. Mean entire pregnancy values for $\mathrm{PM}_{10}, \mathrm{O}_{3}$, and $\mathrm{NO}_{2}$ were $39.7,78.1$, and $16.5 \mu \mathrm{g} / \mathrm{m}^{3}$, respectively, with an average of 0.15 fires $/ \mathrm{km}^{2}$ (Table 1). Mean $\mathrm{PM}_{10}$ concentrations over the entire pregnancy were strongly correlated with $\mathrm{O}_{3}$, moderately so with biomass burning, and weakly with $\mathrm{NO}_{2}$ (Table 2).

We identified in unadjusted models a statistically significant decrease in continuous BW per $10 \mu \mathrm{g} / \mathrm{m}^{3}$ increase in $\mathrm{PM}_{10}$ concentrations only during trimester one, but in each trimester and the entire pregnancy period for biomass
Table 2 Spearman rank correlations of mean pollutant concentrations, number of fires per unit area, and the heat index for the entire pregnancy period.

\begin{tabular}{lrrrrr}
\hline Pollutant & $\mathrm{PM}_{10}$ & \multicolumn{1}{l}{$\mathrm{O}_{3}$} & $\mathrm{NO}_{2}$ & No. of fires & Heat index \\
\hline $\mathrm{PM}_{10}$ & 1.00 & & & & \\
$\mathrm{O}_{3}$ & 0.74 & 1.00 & & & \\
$\mathrm{NO}_{2}$ & 0.37 & 0.36 & 1.00 & & \\
No. of fires & 0.63 & 0.77 & -0.13 & 1.00 & \\
Heat index & -0.03 & -0.11 & 0.51 & -0.26 & 1.00 \\
\hline
\end{tabular}

burning. These relationships, however, were attenuated to non-significant levels once potentially confounding variables were included in the model. The additional adjustment for mean $\mathrm{NO}_{2}$ levels resulted in a statistically significant decrease in $\mathrm{BW}$ associated with both the entire pregnancy mean concentration of $\mathrm{PM}_{10}$ and levels of biomass burning (Table 3 and Fig. 3). Additional analyses of effect modification by sex did not appear to show any obvious differential effects for males and females for either $\mathrm{PM}_{10}$ or biomass burning exposure (Table S2).

The adjusted logistic regression analysis suggested a reduced risk of LBW per $10 \mu \mathrm{g} / \mathrm{m}^{3}$ increment of $\mathrm{PM}_{10}$ in trimester one. This association maintained after adjustment for $\mathrm{NO}_{2}$ levels; mean $\mathrm{PM}_{10}$ concentrations in trimester two also reached statistical significance in this model. Conversely, unadjusted models for biomass burning indicated an increased risk of LBW; however, these associations were attenuated once adjusted for potential confounders and also for $\mathrm{NO}_{2}$ (Table 4). Additional analyses to assess the presence of interaction of $\mathrm{PM}_{10}$ across sex on LBW showed several significant associations of reduced risk in males and females for exposure in different trimesters. For exposure to biomass burning, there was an increased risk only for trimester three exposure in males after adjustment for $\mathrm{NO}_{2}$ (Table S3).

\section{Discussion}

We present findings of the association between pregnant women's exposure to ambient $\mathrm{PM}_{10}$ and biomass burning in several provinces in Thailand and BW as a continuous and dichotomous $(<2500 \mathrm{~g})$ outcome. Although there have been a number of previous studies on the impact of PM exposure on BW [3, 4], the current research represents one of the few studies in a low- to middle-income country (LMIC) [37] and, additionally, where women are routinely exposed to biomass burning derived PM. Overall, we identified a weak association between both whole pregnancy $\mathrm{PM}_{10}$ and biomass burning exposure and reduced $\mathrm{BW}$ as a continuous measure, but only after adjustment for $\mathrm{NO}_{2}$. There was little evidence 
Table 3 Change in birth weight (in grams with $95 \%$ confidence intervals) associated with a 10 $\mu \mathrm{g} / \mathrm{m}^{3}$ increase in $\mathrm{PM}_{10}$ and 1 standard deviation increase in biomass burning (bold results are statistically significant).

\begin{tabular}{|c|c|c|c|}
\hline Exposure & Model 1 & Model 2 & Model 3 \\
\hline \multicolumn{4}{|l|}{$\mathrm{PM}_{10}$} \\
\hline Trimester 1 & $-2.90(-4.90$ to -0.89$)$ & -0.19 ( -2.93 to 2.56$)$ & $-0.80(-4.12$ to 2.51$)$ \\
\hline Trimester 2 & $-0.86(-2.47$ to 0.75$)$ & $2.21(-0.65$ to 5.08$)$ & $2.28(-1.21$ to 5.76$)$ \\
\hline Trimester 3 & $0.56(-1.57$ to 2.69$)$ & $2.33(-0.54$ to 5.19$)$ & $0.69(-2.78$ to 4.16$)$ \\
\hline Entire pregnancy & $-3.51(-7.09$ to 0.07$)$ & $-2.39(-6.94$ to 2.16$)$ & $-6.81(-12.52$ to -1.10$)$ \\
\hline \multicolumn{4}{|l|}{ Biomass burning } \\
\hline Trimester 1 & $-9.64(-12.51$ to -6.77$)$ & $-0.54(-4.62$ to 3.55$)$ & $-1.39(-5.83$ to 3.06$)$ \\
\hline Trimester 2 & $-7.08(-9.84$ to -4.31$)$ & $0.77(-3.29$ to 4.83$)$ & $-1.71(-6.32$ to 2.91$)$ \\
\hline Trimester 3 & $-6.01(-8.88$ to -3.15$)$ & $1.48(-2.54$ to 5.49$)$ & $-1.87(-6.54$ to 2.79$)$ \\
\hline Entire pregnancy & $-12.37(-15.13$ to -9.61$)$ & $-3.12(-7.42$ to 1.17$)$ & $-6.34(-11.35$ to -1.34$)$ \\
\hline
\end{tabular}

Model 1 = unadjusted.

Model 2 = adjusted for sex, gravidity, maternal age, gestation age, year, province, heat index.

Model $3=$ model $2+\mathrm{NO}_{2}$.
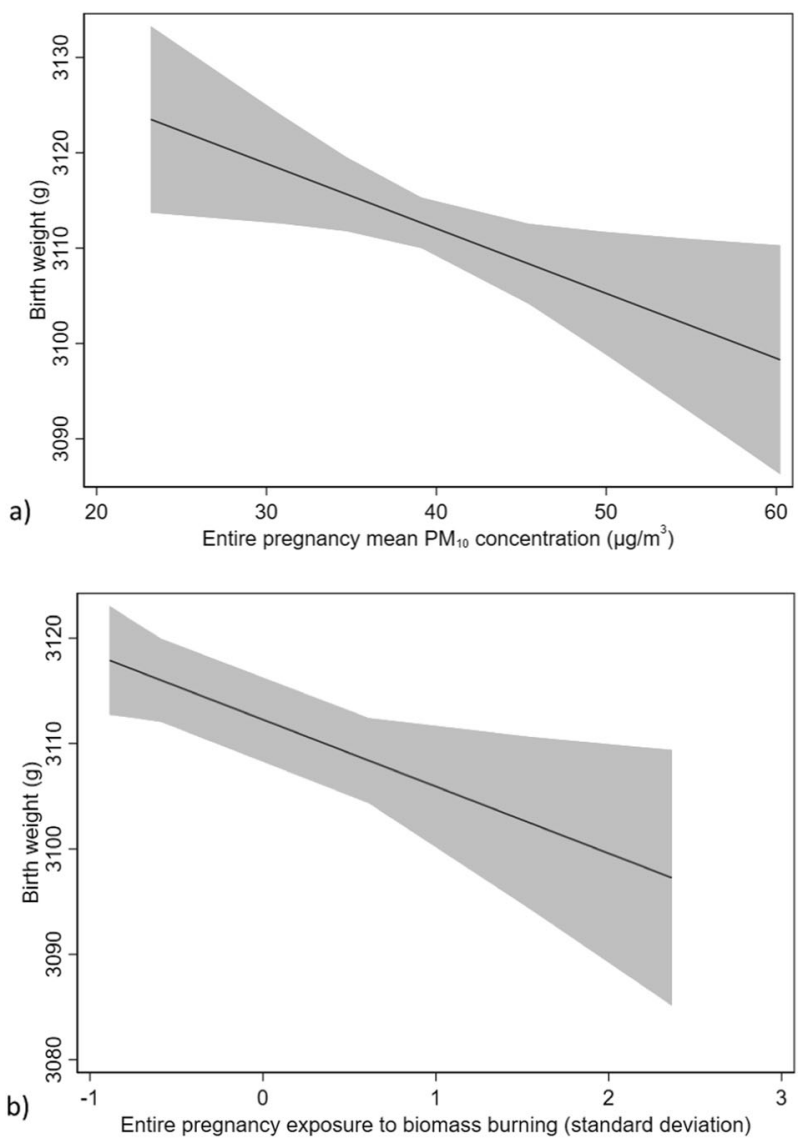

Fig. 3 The average birth weight $(\mathrm{g})$ associated with mean whole pregnancy concentrations of $\mathbf{a} \mathbf{P M}_{10}$ and $\mathbf{b}$ biomass burning across the 1st to 99th percentile of exposure, adjusted for sex, gravidity, maternal age, gestation age, year, province, heat index, and $\mathrm{NO}_{2}$ levels.

of a heightened risk of these exposures with LBW (limited to trimester three exposure to biomass burning in male births); instead, some associations indicated reduced risks with $\mathrm{PM}_{10}$. We discuss our results in relation to previous studies, with special emphasis on those conducted in Asia.

\section{Continuous BW}

In the fully adjusted models examining continuous BW as an outcome, we identified a risk associated with $\mathrm{PM}_{10}$ concentrations and biomass burning during the entire pregnancy, but only when adjusting for $\mathrm{NO}_{2}$ levels. We did not identify in adjusted models a risk of lower BW in connection with such exposures during individual trimesters. Previous studies of BW in Asia have identified statistical associations between PM exposure and reduced BW, but the timing and risk magnitude have varied. For example, studies have found higher risks with $\mathrm{PM}$ exposure in trimesters one ( $\left.\mathrm{PM}_{7}[38]\right)$, two $\left(\mathrm{PM}_{10}\right.$ [39]), and three $\left(\mathrm{PM}_{2.5}\right.$ [34]), while Balakrishnan et al. [40] and Xiao et al. [41] found lower $\mathrm{BW}$ only with $\mathrm{PM}_{2.5}$ concentrations during the entire pregnancy. None of these studies included $\mathrm{PM}_{10}$ risk estimates that were adjusted for the presence of other pollutants. Inconsistencies in potential critical windows of exposure also have been observed across the broader (i.e., outside of Asia) literature [4], as well as in the few studies examining exposure to biomass burning or wildfires. Abdo et al. [42] identified lower BW from exposure to wildfire $\mathrm{PM}_{2.5}$ in Colorado, USA only during the first trimester, while Holstius et al. [43] found the largest decreases in BW when exposure to wildfires in California, USA occurred during the second trimester; decreases in the first trimester were not statistically significant.

Meta-analyses of single-pollutant models of whole pregnancy exposure to $\mathrm{PM}_{10}$ have found reductions in $\mathrm{BW}$ ranging from $-2.7 \mathrm{~g}$ (95\% CI: -7.2 to 1.7$)$ [44] to $-8.4 \mathrm{~g}(95 \% \mathrm{CI}$ : -10.1 to -6.7 ) [9] per $10 \mu \mathrm{g} / \mathrm{m}^{3}$ increase in $\mathrm{PM}_{10}$. As suggested by the positive upper confidence interval in the former, not all research has found BW reductions with coarser PM fractions. In the present study, the effect estimate of the fully adjusted single-pollutant (entire pregnancy) model ( $-2.4 \mathrm{~g}$ per $10 \mu \mathrm{g} / \mathrm{m}^{3}$ [95\% CI: -6.9 to 2.2$]$ ) was quite close in magnitude 
Table 4 Logistic regression model results (odds ratios with 95\% confidence intervals) of $\mathrm{PM}_{10}\left(\right.$ per $\left.10 \mu \mathrm{g} / \mathrm{m}^{3}\right)$ and biomass burning (per 1 standard deviation) exposure with lowbirth weight $(<2500 \mathrm{~g})$ (bold results are statistically significant).

\begin{tabular}{|c|c|c|c|}
\hline Exposure & Model 1 & Model 2 & Model 3 \\
\hline \multicolumn{4}{|l|}{$\mathrm{PM}_{10}$} \\
\hline Trimester 1 & $0.993(0.971-1.015)$ & $0.963(0.933-0.993)$ & $0.953(0.917-0.991)$ \\
\hline Trimester 2 & $1.005(0.988-1.023)$ & $0.968(0.936-1.001)$ & $0.942(0.905-0.981)$ \\
\hline Trimester 3 & $0.984(0.961-1.007)$ & $0.973(0.942-1.006)$ & $0.977(0.939-1.017)$ \\
\hline Entire pregnancy & $0.996(0.957-1.036)$ & $0.987(0.937-1.040)$ & $0.964(0.902-1.030)$ \\
\hline \multicolumn{4}{|l|}{ Biomass burning } \\
\hline Trimester 1 & $1.024(0.993-1.057)$ & $0.981(0.936-1.028)$ & $0.980(0.931-1.032)$ \\
\hline Trimester 2 & $1.034(1.004-1.065)$ & $0.993(0.948-1.040)$ & $0.990(0.939-1.044)$ \\
\hline Trimester 3 & $1.033(1.002-1.065)$ & $1.014(0.968-1.061)$ & $1.033(0.979-1.089)$ \\
\hline Entire pregnancy & $1.049(1.018-1.080)$ & $1.017(0.969-1.068)$ & $1.012(0.956-1.072)$ \\
\hline
\end{tabular}

Model 1 = unadjusted.

Model 2 = adjusted for sex, gravidity, maternal age, gestation age, year, province, heat index.

Model $3=\operatorname{model} 2+\mathrm{NO}_{2}$. to that of Dadvand et al. [44]. More recently, Li et al. [45] did not find any association with $\mathrm{BW}$ and $\mathrm{PM}_{10}$ exposure in Ningbo, China (but did identify reductions with $\mathrm{PM}_{2.5}$ ). Interestingly, that study identified higher $\mathrm{BW}$ with $\mathrm{NO}_{2}$ exposure, but did not offer an explanation for this observation. In our study, we found lower $\mathrm{BW}$ with $\mathrm{PM}_{10}$ and biomass burning occurred only when $\mathrm{NO}_{2}$ concentrations were taken into account, which could occur in the presence of a positive association between $\mathrm{NO}_{2}$ and $\mathrm{BW}$. A possible explanation of this trend might be that $\mathrm{NO}_{2}$ exposures were confounded by the degree of urbanicity: rural areas likely have both lower ambient $\mathrm{NO}_{2}$ concentrations [46] and lower BWs [47]. We did not have maternal residential addresses with birth records, so could not control for urban/rural factors in our analysis.

Our results when examining the effect of $\mathrm{PM}_{10}$ and biomass burning exposure by sex were mostly consistent with the main analysis, though there were slightly greater decreases associated with biomass burning exposure in females (Table S2). Balakrishnan et al. [40] found a statistically significant decrease in continuous BW only for females (associated with $\mathrm{PM}_{2.5}$ ), which was also identified by Merklinger-Gruchala and Kapiszewska [48] for whole pregnancy $\mathrm{PM}_{10}$ exposures. Bell et al. [49] found an adverse association for both male and female infants, though with slightly greater effects in females. In contrast to these findings, O'Donnell and Behie [50] discovered heavier male infants born to mothers exposed to wildfire smoke in Australia. Nevertheless, the point estimates of entire pregnancy exposures for males and females identified in the present study were comparable in magnitude and with most previous findings (i.e., $<10 \mathrm{~g}$ per $10 \mu \mathrm{g} / \mathrm{m}^{3}$ ).

\section{Low birth weight}

While BW as a continuous variable was lower on average with entire pregnancy $\mathrm{PM}_{10}$ concentrations and biomass burning in the presence of $\mathrm{NO}_{2}$, there was a reduced risk of LBW with $\mathrm{PM}_{10}$ exposure in trimesters one and two; no statistical relationships were apparent among the full pregnancy period for either $\mathrm{PM}_{10}$ or biomass burning. Unlike results with the continuous measure, this finding was consistent both with and without $\mathrm{NO}_{2}$, although only in the first trimester. ORs reported in meta-analyses of LBW with entire pregnancy $\mathrm{PM}_{10}$ per $10 \mu \mathrm{g} / \mathrm{m}^{3}$ are modest, ranging from 1.01 (95\% CI: 0.96-1.08) [8] to 1.05 (95\% CI: 1.02-1.07) [9], and those with separate analyses for Asian countries $[8,51]$ show ORs not significantly different from 1.00 for LBW with $\mathrm{PM}_{10}$ concentrations. As in the present research, some past studies have found a reduced risk of LBW with $\mathrm{PM}_{10}$ exposures, based on land use regression models and ground monitoring data in Brazil $[52,53]$, trimester one $\mathrm{PM}_{10}$ exposures assigned from monitors in China [54], and whole pregnancy $\mathrm{PM}_{2.5}$ exposures in Canada based on satellite derived estimates [55]. Habermann and Gouveia [52] showed that in Sao Paulo, Brazil, higher socioeconomic status (SES) was correlated with higher whole pregnancy air pollution exposures; therefore, some of the positive association of SES on BW could have been captured by air pollution indicators in their model. Our study found a protective effect on LBW for exposure in trimester one in both single and multipollutant models. As discussed in Fleischer et al. [56], it is possible that the foetus may not survive high exposures during critical periods in gestation. If so, an apparent protective effect in live births of exposure to air pollution might mask any such detrimental effect. There is only weak evidence available to support this hypothesis: Hwang et al. [57] found increased stillbirths in Taiwan with $\mathrm{PM}_{10}$ concentrations in gestation months one and two, coinciding with lowered risk estimates observed in trimester one in the present research. Despite these protective associations, our null findings for the entire pregnancy are aligned with findings documented in several cohort studies [4]. 
In adjusted single-pollutants models, we found a statistically significant protective effect for males with $\mathrm{PM}_{10}$ exposure in trimester one and females with trimester three exposures, with no effects for either sex observed for the whole pregnancy. This contrasts with Balakrishnan et al. [40], who found a statistically significant increased risk only for female births and entire pregnancy $\mathrm{PM}_{2.5}$. A review based on limited studies found that males might be more susceptible to risks from air pollution due to being less mature at term [58]. Our findings of protective effects with each sex are at odds with this earlier work. A study of biomass burning in Brazil found an $\sim 50 \%$ increase in LBW in the highest quartile of $\mathrm{PM}_{2.5}$ exposure for trimesters two and three [59]. Our finding of an increased risk in LBW for males and trimester three biomass burning exposure is somewhat consistent with these findings; however, our effect estimate was more modest, and the previous study did not examine sex differences. Further to the discussion above on this topic, these findings of possible effect modification might be attributed to some unmeasured confounder, exposure misclassification, or spurious associations, ultimately suggesting an unclear and/or seemingly weak overall effect of $\mathrm{PM}_{10}$ on LBW.

\section{Strengths and limitations of the study}

A major strength of our study was the availability of detailed air pollutant and biomass burning data with individual birth records from a LMIC in a tropical setting. We were able to adjust for some potential maternal confounders, including age and the number of prior pregnancies. Nevertheless, we did not have information on other potentially important confounding variables, such as SES, maternal cigarette smoking, indoor air pollution, and malarial infection. Although the proportion of female smokers is low in Thailand ( 2\% [60]), the foetus still may have been exposed to second-hand smoke, which would have had a detrimental effect on BW [61]. Likewise, we did not account for indoor air pollution, which has been shown to have adverse effects with birth outcomes [62], nor did we account for different sources of PM. Malarial infections during pregnancy may also influence $\mathrm{BW}$, but there were only about 100 cases of malaria reported during the study period [63], so it is not likely to have had a substantial impact on our results. Other behaviours (e.g., alcohol consumption) that were not accounted for in our analysis and do not differ greatly by season are not likely to have confounded our findings [2]. There was evidence in our dataset that BWs were being rounded to the nearest $10 \mathrm{~g}$ and, less commonly, $100 \mathrm{~g}$, which has been previously reported in both LMICs [64] and higher income countries [65] to various degrees. This trend, assuming no bias in the direction of rounding, would introduce imprecision to risk estimates or, more extremely, lead to the inability to detect an effect.
Therefore, rounding might have attenuated the estimates in the present study for the continuous birth outcome. In addition, we were not able to exclude multiple births, which, as with rounding, might have attenuated statistical associations. Ultimately, even assuming a significant decreased association between PM and BW, there is still too much uncertainty to quantify health risks later in life based on the magnitude of observed BW reductions (i.e., $\sim 10 \mathrm{~g}$ ) [6], in part because studies tend to examine such risks on a much larger scale (e.g., per $1 \mathrm{~kg}$ increment) [66].

We used ground monitors to assign exposure, which are a somewhat crude indicator of air pollution levels and may not capture important spatial differences in exposure within smaller areas [67]. Further, this source of exposure data may be more problematic when comparing particles and reactive gases, such as $\mathrm{NO}_{2}$ and $\mathrm{O}_{3}$; gases may exhibit different spatial and temporal distributions [68]. Further, we did not have sufficient $\mathrm{PM}_{2.5}$ data to examine associations with BW, which may have produced different findings. Monitors in Thailand, as elsewhere, tend to be situated in more urban or other areas of higher ambient concentrations for regulatory purposes [69]. Thus, exposures assigned to each BW in our study might have been inflated, which would have lessened the magnitude of any resulting air pollution risk estimates (similar to the aforementioned rounding effect). In contrast with the spatially limited $\mathrm{PM}_{10}$ exposure data, our exposure metric for biomass burning included fires from across each province; the similarity of results among $\mathrm{PM}_{10}$ and biomass burning with continuous $\mathrm{BW}$ therefore may underscore the importance of temporal over spatial variability in exposures. Due to the high correlations observed in our study between pregnancy exposure levels of $\mathrm{PM}_{10}$ and $\mathrm{O}_{3}$, we were unable to distinguish PM effects from $\mathrm{O}_{3}$ on $\mathrm{BW}$. These elevated ambient $\mathrm{O}_{3}$ concentrations may have occurred downwind from biomass burning [70].

\section{Conclusion}

We present here one of the few studies examining air pollution and $\mathrm{BW}$ in a tropical LMIC where biomass burning is widespread and an important source of PM emissions. Our findings suggest a potential association between exposure to ambient $\mathrm{PM}_{10}$ concentrations and biomass burning with reduced $\mathrm{BW}$ as a continuous measure; however, there was little indication of a clear relationship with LBW (i.e., $<2500 \mathrm{~g}$ ). Although our findings contribute to the relatively small evidence base on the maternal health effects from short- to medium-term ambient air pollution events [18], our study is based on province-level $\mathrm{PM}_{10}$ exposures and should be refined in future research to better account for spatial variability and to include exposure to $\mathrm{PM}_{2.5}$. While much of the biomass burning occurs in the north of 
Thailand, PM can travel long distances and has been documented in Bangkok [71]; thus, these potentially harmful exposures are not localised. As populations in LMICs typically are exposed to higher PM levels, the evidence base in these areas in particular should be expanded to help inform policy efforts of air pollution reduction.

Acknowledgements We acknowledge the use of data and imagery from LANCE FIRMS operated by NASA's Earth Science Data and Information System (ESDIS) with funding provided by NASA Headquarters. This study was funded by the Medical Research Council (MRC) (MR/R006210/1) and the Thailand Research Fund (TRF) (RDG603009). Funds to support open access were provided by HeriotWatt University. The views expressed are those of the author(s) and not necessarily those of the MRC or TRF.

\section{Compliance with ethical standards}

Conflict of interest The authors declare no competing interests.

Publisher's note Springer Nature remains neutral with regard to jurisdictional claims in published maps and institutional affiliations.

Open Access This article is licensed under a Creative Commons Attribution 4.0 International License, which permits use, sharing, adaptation, distribution and reproduction in any medium or format, as long as you give appropriate credit to the original author(s) and the source, provide a link to the Creative Commons license, and indicate if changes were made. The images or other third party material in this article are included in the article's Creative Commons license, unless indicated otherwise in a credit line to the material. If material is not included in the article's Creative Commons license and your intended use is not permitted by statutory regulation or exceeds the permitted use, you will need to obtain permission directly from the copyright holder. To view a copy of this license, visit http://creativecommons. org/licenses/by/4.0/

\section{References}

1. Liu Y, Xu J, Chen D, Sun P, Ma X. The association between air pollution and preterm birth and low birth weight in Guangdong, China. BMC Public Health. 2019;19:3.

2. Klepac P, Locatelli I, Korošec S, Künzli N, Kukec A. Ambient air pollution and pregnancy outcomes: A comprehensive review and identification of environmental public health challenges. Environ Res. 2018;167:144-159.

3. Li X, Huang S, Jiao A, Yang X, Yun J, Wang Y, et al. Association between ambient fine particulate matter and preterm birth or term low birth weight: an updated systematic review and meta-analysis. Environ Pollut. 2017;227:596-605.

4. Yuan L, Zhang Y, Gao Y, Tian Y. Maternal fine particulate matter (PM 2.5) exposure and adverse birth outcomes: an updated systematic review based on cohort studies. Environ Sci Pollut Res. 2019;26:13963-13983.

5. Proietti E, Röösli M, Frey U, Latzin P. Air pollution during pregnancy and neonatal outcome: a review. J Aerosol Med Pulm Drug Deliv. 2013;26:9-23.

6. Steinle S, Johnston HJ, Loh M, Mueller W, Vardoulakis S, Tantrakarnapa K, et al. In utero exposure to particulate air pollution during pregnancy: impact on birth weight and health through the life course. Int $\mathrm{J}$ Environ Res Public Health. 2020;17:8948.
7. Blanc AK, Wardlaw T. Monitoring low birth weight: an evaluation of international estimates and an updated estimation procedure. Bull World Health Organ. 2005;83:178-185d.

8. Ji Y, Song F, Xu B, Zhu Y, Lu C, Xia Y. Association between exposure to particulate matter during pregnancy and birthweight: a systematic review and a meta-analysis of birth cohort studies. J Biomed Res. 2019;33:56.

9. Stieb DM, Chen L, Eshoul M, Judek S. Ambient air pollution, birth weight and preterm birth: a systematic review and metaanalysis. Environ Res. 2012;117:100-111.

10. Chaudhuri I, Fruijtier-Pölloth C, Ngiewih Y, Levy L. Evaluating the evidence on genotoxicity and reproductive toxicity of carbon black: a critical review. Crit Rev Toxicol. 2018;48:143-169.

11. Ema M, Naya M, Horimoto M, Kato H. Developmental toxicity of diesel exhaust: a review of studies in experimental animals. Reprod Toxicol. 2013;42:1-17.

12. Kannan S, Misra DP, Dvonch JT, Krishnakumar A. Exposures to airborne particulate matter and adverse perinatal outcomes: a biologically plausible mechanistic framework for exploring potential effect modification by nutrition. Environ Health Perspect. 2006;114:1636-1642.

13. Chen G, Guo Y, Abramson MJ, Williams G, Li S. Exposure to low concentrations of air pollutants and adverse birth outcomes in Brisbane, Australia, 2003-2013. Sci Total Environ. 2018;622:721-726.

14. Wang Q, Liang Q, Li C, Ren M, Lin S, Knibbs LD, et al. Interaction of air pollutants and meteorological factors on birth weight in Shenzhen, China. Epidemiology. 2019;30:S57-S66.

15. Cottrell G, Mary JY, Barro D, Cot M. The importance of the period of malarial infection during pregnancy on birth weight in tropical Africa. Am J Tropical Med Hyg. 2007;76:849-854.

16. Johnston HJ, Mueller W, Steinle S, Vardoulakis S, Tantrakarnapa $\mathrm{K}$, Loh $\mathrm{M}$, et al. How harmful is particulate matter emitted from biomass burning? A Thailand perspective. Curr Pollut Rep. 2019;5:353-377.

17. Mueller W, Loh M, Vardoulakis S, Johnston HJ, Steinle S, Precha $\mathrm{N}$, et al. Ambient particulate matter and biomass burning: an ecological time series study of respiratory and cardiovascular hospital visits in northern Thailand. Environ Health. 2020;19:1-12.

18. Melody SM, Ford J, Wills K, Venn A, Johnston FH. Maternal exposure to short-to medium-term outdoor air pollution and obstetric and neonatal outcomes: a systematic review. Environ Pollut. 2019;244:915-925.

19. Climates to Travel. n.d. Climate-Thailand. https://www.clima testotravel.com. Last accessed 14 Oct 2020.

20. Central Intelligence Agency (CIA). The World Factbook. 2020. https://www.cia.gov/library/publications/the-world-factbook/geos/ th.html. Last accessed 10 Mar 2020.

21. NASA. Fire Information for Resource Management System (FIRMS). 2020. https://earthdata.nasa.gov/earth-observation-data/ near-real-time/firms. Last accessed 10 Mar 2020

22. Kliengchuay W, Cooper Meeyai A, Worakhunpiset S, Tantrakarnapa K. Relationships between meteorological parameters and particulate matter in Mae Hong Son province, Thailand. Int $\mathbf{J}$ Environ Res Public Health. 2018;15:2801.

23. Uttamang P, Aneja VP, Hanna AF. Assessment of gaseous criteria pollutants in the Bangkok Metropolitan Region, Thailand. Atmos Chem Phys. 2018;18:12581-12593.

24. World Health Organization (WHO). Global health observatory country views. 2019. http://apps.who.int/gho/data/node.country. country-THA. Last accessed 10 Mar 2020.

25. Tangcharoensathien V, Witthayapipopsakul W, Panichkriangkrai W, Patcharanarumol W, Mills A. Health systems development in Thailand: a solid platform for successful implementation of universal health coverage. Lancet. 2018;391:1205-1223. 
26. Witthayapipopsakul W, Kulthanmanusorn A, Vongmongkol V, Viriyathorn S, Wanwong Y, Tangcharoensathien V. Achieving the targets for universal health coverage: how is Thailand monitoring progress? WHO South-East Asia J Public Health. 2019;8:10-17.

27. Swamy GK, Edwards S, Gelfand A, James SA, Miranda ML. Maternal age, birth order, and race: differential effects on birthweight. J Epidemiol Community Health. 2012;66:136-142.

28. Wong CM, Vichit-Vadakan N, Kan H, Qian Z. Public Health and Air Pollution in Asia (PAPA): a multicity study of short-term effects of air pollution on mortality. Environ Health Perspect. 2008;116:1195-1202.

29. Liang L, Cai Y, Barratt B, Lyu B, Chan Q, Hansell AL, et al. Associations between daily air quality and hospitalisations for acute exacerbation of chronic obstructive pulmonary disease in Beijing, 2013-17: an ecological analysis. Lancet Planet Health. 2019;3:e270-e279.

30. Schroeder W, Oliva P, Giglio L, Csiszar IA. The New VIIRS 375 $\mathrm{m}$ active fire detection data product: Algorithm description and initial assessment. Remote Sens Environ. 2014;143:85-96.

31. Hinkle SN, Albert PS, Mendola P, Sjaarda LA, Yeung E, Boghossian NS, et al. The association between parity and birthweight in a longitudinal consecutive pregnancy cohort. Paediatr Perinat Epidemiol. 2014;28:106-115.

32. Hughes MM, Katz J, Mullany LC, Khatry SK, LeClerq SC, Darmstadt GL, et al. Seasonality of birth outcomes in rural Sarlahi District, Nepal: a population-based prospective cohort. BMC Pregnancy Childbirth. 2014;14:1-9.

33. Anderson GB, Bell ML, Peng RD. Methods to calculate the heat index as an exposure metric in environmental health research. Environ Health Perspect. 2013;121:1111-1119.

34. Rich DQ, Liu K, Zhang J, Thurston SW, Stevens TP, Pan Y, et al. Differences in birth weight associated with the 2008 Beijing Olympics air pollution reduction: results from a natural experiment. Environ Health Perspect. 2015;123:880-887.

35. Department for Environment, Food \& Rural Affairs (DEFRA). Conversion Factors Between ppb and $\mu \mathrm{g} \mathrm{m}^{-3}$ and $\mathrm{ppm}$ and $\mathrm{mgm}^{-3}$. https:/uk-air.defra.gov.uk/assets/documents/reports/cat06/ 0502160851_Conversion_Factors_Between_ppb_and.pdf. Last accessed 10 March 2020.

36. Wilson A, Chiu YHM, Hsu HHL, Wright RO, Wright RJ, Coull BA. Potential for bias when estimating critical windows for air pollution in children's health. Am J Epidemiol. 2017; 186:1281-1289.

37. Mannucci PM, Franchini M. Health effects of ambient air pollution in developing countries. Int J Environ Res Public Health. 2017; $14: 1048$.

38. Yorifuji T, Kashima S, Doi H. Outdoor air pollution and term low birth weight in Japan. Environ Int. 2015;74:106-111.

39. Han Y, Ji Y, Kang S, Dong T, Zhou Z, Zhang Y, et al. Effects of particulate matter exposure during pregnancy on birth weight: a retrospective cohort study in Suzhou, China. Sci Total Environ. 2018;615:369-374.

40. Balakrishnan K, Ghosh S, Thangavel G, Sambandam S, Mukhopadhyay K, Puttaswamy N, et al. Exposures to fine particulate matter (PM2. 5) and birthweight in a rural-urban, mother-child cohort in Tamil Nadu, India. Environ Res. 2018;161:524-531.

41. Xiao Q, Chen H, Strickland MJ, Kan H, Chang HH, Klein M, et al. Associations between birth outcomes and maternal PM2. 5 exposure in Shanghai: a comparison of three exposure assessment approaches. Environ Int. 2018;117:226-236.

42. Abdo M, Ward I, O'Dell K, Ford B, Pierce JR, Fischer EV, et al. Impact of wildfire smoke on adverse pregnancy outcomes in Colorado, 2007-2015. Int J Environ Res Public Health. 2019;16:3720.
43. Holstius DM, Reid CE, Jesdale BM, Morello-Frosch R. Birth weight following pregnancy during the 2003 Southern California wildfires. Environ health Perspect. 2012;120:1340-1345.

44. Dadvand P, Parker J, Bell ML, Bonzini M, Brauer M, Darrow LA, et al. Maternal exposure to particulate air pollution and term birth weight: a multi-country evaluation of effect and heterogeneity. Environ Health Perspect. 2013;121:267-373.

45. Li Z, Yuan X, Fu J, Zhang L, Hong L, Hu L, et al. Association of ambient air pollutants and birth weight in Ningbo, 2015-2017. Environ Pollut. 2019;249:629-637.

46. Bootdee S, Chalemrom P, Chantara S. Validation and field application of tailor-made nitrogen dioxide passive samplers. Int $\mathrm{J}$ Environ Sci Technol. 2012;9:515-526.

47. Kaur S, Ng CM, Badon SE, Jalil RA, Maykanathan D, Yim HS, et al. Risk factors for low birth weight among rural and urban Malaysian women. BMC Public Health. 2019;19:539.

48. Merklinger-Gruchala A, Kapiszewska M. Association between PM10 air pollution and birth weight after full-term pregnancy in Krakow city 1995-2009-trimester specificity. Ann Agric Environ Med. 2015;22:265-270.

49. Bell ML, Ebisu K, Belanger K. The relationship between air pollution and low birth weight: effects by mother's age, infant sex, co-pollutants, and pre-term births. Environ Res Lett. 2008;3:044003.

50. O'Donnell MH, Behie AM. Effects of wildfire disaster exposure on male birth weight in an Australian population. Evolution Med Public Health. 2015;2015:344-354.

51. Guo LQ, Chen Y, Mi BB, Dang SN, Zhao DD, Liu R, et al. Ambient air pollution and adverse birth outcomes: A systematic review and meta-analysis. $J$ Zhejiang Univ-Sci B. 2019;20:238-252.

52. Habermann M and Gouveia N. Socioeconomic position and low birth weight among mothers exposed to traffic-related air pollution. PLoS ONE. 2014;9:e113900.

53. Nascimento LFC, Machin AB, Santos DAAD. Are there differences in birth weight according to sex and associations with maternal exposure to air pollutants? A cohort study. Sao Paulo Med J. 2017;135:347-354.

54. Ye L, Ji Y, Lv W, Zhu Y, Lu C, Xu B, et al. Associations between maternal exposure to air pollution and birth outcomes: a retrospective cohort study in Taizhou, China. Environ Sci Pollut Res. 2018;25:21927-21936.

55. Lavigne E, Yasseen AS III, Stieb DM, Hystad P, Van Donkelaar A, Martin RV, et al. Ambient air pollution and adverse birth outcomes: differences by maternal comorbidities. Environ Res. 2016;148:457-466.

56. Fleischer NL, Merialdi M, van Donkelaar A, Vadillo-Ortega F, Martin RV, Betran AP, et al. Outdoor air pollution, preterm birth, and low birth weight: analysis of the world health organization global survey on maternal and perinatal health. Environ Health Perspect. 2014;122:425-430.

57. Hwang BF, Lee YL, Jaakkola JJ. Air pollution and stillbirth: a population-based case-control study in Taiwan. Environ Health Perspect. 2011;119:1345-1349.

58. Ghosh R, Rankin J, Pless-Mulloli T, Glinianaia S. Does the effect of air pollution on pregnancy outcomes differ by gender? A systematic review. Environ Res. 2007;105:400-408.

59. da Silva AMC, Moi GP, Mattos IE, de Souza Hacon S. Low birth weight at term and the presence of fine particulate matter and carbon monoxide in the Brazilian Amazon: a population-based retrospective cohort study. BMC Pregnancy Childbirth. 2014;14:309.

60. Aungkulanon S, Pitayarangsarit S, Bundhamcharoen K, Akaleephan $\mathrm{C}$, Chongsuvivatwong V, Phoncharoen R, et al. Smoking prevalence and attributable deaths in Thailand: predicting outcomes of different tobacco control interventions. BMC Public Health. 2019;19:984. 
61. Khader YS, Al-Akour N, AlZubi IM, Lataifeh I. The association between second hand smoke and low birth weight and preterm delivery. Matern Child Health J. 2011;15:453-459.

62. Pope DP, Mishra V, Thompson L, Siddiqui AR, Rehfuess EA, Weber M, et al. Risk of low birth weight and stillbirth associated with indoor air pollution from solid fuel use in developing countries. Epidemiol Rev. 2010;32:70-81.

63. Ministry of Public Health. 2020. Malaria map. http://mgis.ddc. moph.go.th/. Last accessed: 9 Mar 2020.

64. Channon AA, Padmadas SS, McDonald JW. Measuring birth weight in developing countries: does the method of reporting in retrospective surveys matter? Matern Child Health J. 2011;15:12-18.

65. Emmerson AJ, Roberts SA. Rounding of birth weights in a neonatal intensive care unit over 20 years: an analysis of a large cohort study. BMJ Open. 2013;3:e003650.

66. Belbasis L, Savvidou MD, Kanu C, Evangelou E, Tzoulaki I. Birth weight in relation to health and disease in later life: an umbrella review of systematic reviews and meta-analyses. BMC Med. 2016;14:147.
67. Pedersen M, Giorgis-Allemand L, Bernard C, Aguilera I, Andersen AMN, Ballester F, et al. Ambient air pollution and low birthweight: a European cohort study (ESCAPE). lancet Respiratory Med. 2013;1:695-704.

68. Xie Y, Zhao B, Zhang L, Luo R. Spatiotemporal variations of PM2. 5 and PM10 concentrations between 31 Chinese cities and their relationships with $\mathrm{SO} 2, \mathrm{NO} 2, \mathrm{CO}$ and $\mathrm{O} 3$. Particuology. 2015;20:141-149.

69. Jerrett M, Arain A, Kanaroglou P, Beckerman B, Potoglou D, Sahsuvaroglu T, et al. A review and evaluation of intraurban air pollution exposure models. J Exposure Sci Environ Epidemiol. 2005;15:185-204.

70. Jaffe DA, Wigder NL. Ozone production from wildfires: a critical review. Atmos Environ. 2012;51:1-10.

71. Narita D, Oanh NTK, Sato K, Huo M, Permadi DA, Chi NNH, et al. Pollution characteristics and policy actions on fine particulate matter in a growing asian economy: the case of Bangkok Metropolitan Region. Atmosphere. 2019;10:227. 